\title{
Der Energie-Impulsvektor in der unitären divergenzfreien Quantenelektrodynamik •
}

\author{
Von Hermann Kümmel \\ Aus dem Institut für theoretische Physik der Freien Universität Berlin \\ (Z. Naturforschg. 5 a, 642-644 [1950]; eingegangen am 27. November 1950)
}

\begin{abstract}
Es wird ein Energie-Impulsvektor aus dem klassischen, von Whe eler und Feynm a ${ }^{1}$ angegebenen Ausdruck abgeleitet, der in der nach $\mathrm{Lu} \mathrm{d}$ wig ${ }^{2}$ formulierten unitären Quantenelektrodynamik (ohne Löchertheorie) eine verschwindende Selbstenergie liefert.
\end{abstract}

Ludwi g ${ }^{2}$ hat, ausgehend von der Definition ${ }^{3}$

$$
\psi\left(x_{1}\right)=\psi_{0}\left(x_{1}\right)+e^{2} \int K\left(x_{1}-x_{2}\right) \gamma_{\mu} \bar{D}\left(x_{2}-x_{3}\right) \bar{\psi}\left(x_{3}\right) \gamma_{\mu} \psi\left(x_{3}\right) \psi\left(x_{2}\right) \mathrm{d} x_{2} \mathrm{~d} x_{3}
$$

für den $\psi$-Operator eine unitäre Quantenelektrodynamik vorgeschlagen. Hier ist $\psi_{0}$ der gewöhnliche wechselwirkungsfreie Operator des Elektronenwellenfeldes:

$$
\left(\frac{\partial}{\partial x_{v}} \gamma_{v}+m\right) \psi_{0}(x)=0
$$

mit den üblichen Vertauschungsrelationen und $K\left(x_{1}-x_{2}\right)$ ist eine Greensche Funktion der DiracGleichung:

$$
\left(\frac{\partial}{\partial x_{v}} \gamma_{v}+m\right) K\left(x_{1}-x_{2}\right)=-\delta\left(x_{1}-x_{2}\right)
$$

Das Viererpotential ist hierbei weiter nichts als ein Symbol für

$$
A_{v}\left(x_{1}\right)=i e \int \bar{D}\left(x_{1}-x_{2}\right) \bar{\psi}\left(x_{2}\right) \gamma_{v} \psi\left(x_{2}\right) \mathrm{d} x_{2} .
$$

Die Integralgleichung (1) für $\psi$ hat zur Folge, daß die Vertauschungsrelationen gegenüber der üblichen Theorie sich wesentlich ändern: Es ist keineswegs immer $\left[\psi\left(x_{1}\right), \psi\left(x_{2}\right)\right]=0, \ldots,\left[A\left(x_{1}\right), \psi\left(x_{2}\right)\right]=0$; auch nicht, wenn die Punkte raumartig zueinander liegen ${ }^{4}$. Daher versagen die herkömmlichen Energie-Impulstensoren, weil es jetzt sehr auf die Reihenfolge von $A$ und $\psi$ ankommt. Man findet leicht, daß z. B. der nach $\mathrm{Schwinger}{ }^{3}$ gebildete Tensor jetzt keine

1 J. A. Whe ele r u. R. P. F e y n m a n, Rev. mod. Physics 21, 425 [1949].

2 G. Lu dwig, Z. Naturforschg. 5a, 637 [1950].

3 Hier haben wir die von $\mathrm{S} \mathrm{ch}$ winge $\mathrm{r}$ benutzten Bezeichnungen gewählt, s. S c h w in ger, Physic. Rev. 74, 1439 [1948] und Physic. Rev. 75, 651 [1949]. Ferner setzen wir $\hbar=1$ und $c=1$.

4 Eine Untersuchung der Vertauschungsrelationen ist im Gange; wegen der Kompliziertheit derselben ist bisher nur sicher, daß die angegebenen Vertauschungsrelationen nicht überall - auch nicht außerhalb des Lichtkegels verschwinden. Für große raumartige Abstände wird man $\left[A\left(x_{1}\right), \psi\left(x_{2}\right)\right]=0$ erwarten. verschwindende Divergenz mehr besitzt ${ }^{5}$. Glücklicherweise gilt aber noch

mit dem Strom $\frac{\partial}{\partial x_{v}} i_{v}(x)=0$

$$
i_{v}(x)=\bar{\psi}(x) \gamma_{v} \psi(x) \text {. }
$$

Dies weist man nach, indem man benutzt, daß die Wellengleichung sich auch jetzt noch in der Form

$$
\frac{\partial}{\partial x_{v}} \psi \gamma_{v}+m \psi-i e A_{v} \gamma_{v} \psi=0
$$

mit (4) und unter Berücksichtigung von (1) und (3) schreiben läßt.

\section{Klassischer Vektor}

Bei dieser Sachlage beschränken wir uns auf die Bestimmung eines Energie-Impulsvektors. Nach Wheeler und Feynman ${ }^{1}$ ist dieser für zwei Elektronen gegeben durch

$$
\begin{aligned}
& G_{m}(a, \beta)=m\left\{\dot{a}_{m}(a)+\dot{b}_{m}(\beta)\right\} \\
& +\frac{1}{2 \pi} e^{2}\left\{-\int_{-\infty}^{a} \int_{\beta}^{\infty}+\int_{a}^{\infty} \int_{-\infty}^{\beta}\right\} \delta^{\prime}\left[(a-b)_{\nu}^{2}\right] \\
& \cdot\left[(a-b)_{m} \mathrm{~d} a_{\mu} \mathrm{d} b_{\mu}-\mathrm{d} b_{m} \mathrm{~d} a_{\mu}(a-b)_{\mu}-\mathrm{d} a_{m} \mathrm{~d} b_{\mu}(a-b)_{\mu}\right]
\end{aligned}
$$

5 Es bleibt - bei der günstigsten Wahl der Reihenfolge - immer noch ein Glied

$$
\begin{gathered}
-\frac{1}{2} \lim _{x_{1}{ }^{\prime} \rightarrow x_{1}} \frac{\partial^{2}}{\partial x_{\mu}^{1} \partial x_{\varrho}^{1}} \int \bar{D}\left(x_{1}-x_{2}\right) \bar{\psi}\left(x_{2}\right) F_{v \varrho}\left(x_{1}{ }^{\prime}\right) \gamma_{\mu} \psi\left(x_{2}\right) \mathrm{d} x_{2} \\
\text { mit } \quad F_{v \varrho}=A_{\varrho \mid v}-A_{v \mid \varrho}
\end{gathered}
$$

übrig, das in der üblichen Theorie wegen $A_{v \mid v} \Phi=0 \mathrm{im}$ betrachteten Hilbert-Raum verschwindet. Wählt man statt (1) eine andere Reihenfolge der Operatoren in der Integralgleichung, so gelingt zwar die Definition eines divergenzfreien Tensors, nur wird dann die Selbstenergie nicht mehr endlich, da dann Glieder der Form $\bar{\psi} \psi \bar{\psi} \psi$ unter dem Energie-Impulsintegral auftreten. 
Hier sind $a, b$ die Weltlinien der beiden betrachteten Elektronen, und $\alpha, \beta$ ihre Eigenzeiten. Wir ersetzen die Punktladungen durch stetige Ladungsverteilungen:

$$
\mathrm{d} a=\frac{1}{e} j(x) \mathrm{d} x
$$

$G_{m}(\sigma)=\int_{\sigma} \bar{\psi} \gamma_{v}\left(\psi_{\mid m}-i e A_{m} \psi\right) \mathrm{d} \sigma_{v}$

$+\frac{1}{4} \frac{1}{4 \pi}\left\{-\int_{-\infty}^{\sigma} \int_{\sigma}^{\infty}+\int_{\sigma}^{\infty} \int_{-\infty}^{\sigma}\right\}\left[\delta_{\mid m}\left[\left(x_{1}-x_{2}\right)_{v}^{2}\right] j_{\mu}\left(x_{1}\right) j_{\mu}\left(x_{2}\right)-\delta_{\mid \mu}\left[\left(x_{1}-x_{2}\right)_{v}^{2}\right]\left(j_{m}\left(x_{1}\right) j_{\mu}\left(x_{2}\right)+j_{m}\left(x_{2}\right) j_{\mu}\left(x_{1}\right)\right)\right] \mathrm{d} x_{1} \mathrm{~d} x_{2}$.

Wegen der Antisymmetrie des Integranden des zweiten Anteiles wird daraus, wenn wir noch

$$
A_{m}=\frac{1}{2}\left[A_{m}^{\text {avanc. }}+A_{m}^{\text {retard. }}\right]
$$

setzen:

$$
\begin{aligned}
G_{m}(\sigma) & =\int_{\sigma} \bar{\psi} \gamma_{v}\left(\psi_{\mid m}-\frac{i e}{2}\left[A_{m}^{\mathrm{av} .}+A_{m}^{\mathrm{ret}}\right] \psi\right) \mathrm{d} \sigma_{v} \\
+ & \frac{1}{2} \frac{1}{4 \pi} \int_{\sigma}^{\infty} \int_{-\infty}^{\sigma}\left[\delta_{\mid m}\left[\left(x_{1}-x_{2}\right)_{v}^{2}\right] j_{\mu}\left(x_{1}\right) j_{\mu}\left(x_{2}\right)-\delta_{\mid \mu}\left[\left(x_{1}-x_{2}\right)_{\nu}^{2}\right]\left(j_{m}\left(x_{1}\right) j_{\mu}\left(x_{2}\right)+i_{m}\left(x_{2}\right) j_{\mu}\left(x_{1}\right)\right)\right] \mathrm{d} x_{1} \mathrm{~d} x_{2} .
\end{aligned}
$$

Von diesem Vektor müssen wir den Energie- und Impulssatz verlangen:

Wegen

$$
\frac{\delta G(\sigma)}{\delta \sigma(x)}=0 .
$$

und der bekannten Divergenzrelation des Materietensors bzw. des Stromes folgt nach kurzer Rechnung die Behauptung (12). Damit ist die im Übergang von (8) zu (9) steckende Willkür gerechtfertigt. (11) läßt sich noch wesentlich vereinfachen.

Es ist

$$
\begin{aligned}
& \int_{\sigma}^{\infty} \int_{-\infty}^{\sigma} \delta_{\mid \mu}\left[\left(x_{1}-x_{2}\right)_{v}^{2}\right]\left[j_{m}\left(x_{2}\right) j_{\mu}\left(x_{1}\right)+j_{\mu}\left(x_{2}\right) j_{m}\left(x_{1}\right)\right] \mathrm{d} x_{1} \mathrm{~d} x_{2} \\
& \quad=\int_{\sigma}^{\infty} \int_{-\infty}^{\sigma}\left[\frac{\partial}{\partial x_{\mu}^{1}}\left(\delta\left[\left(x_{1}-x_{2}\right)_{v}^{2}\right] j_{\mu}\left(x_{1}\right)\right) j_{m}\left(x_{2}\right)-\frac{\partial}{\partial x_{\mu}^{2}}\left(\delta\left[\left(x_{1}-x_{2}\right)_{v}^{2}\right] j_{\mu}\left(x_{2}\right)\right) j_{m}\left(x_{1}\right)\right] \mathrm{d} x_{1} \mathrm{~d} x_{2} \\
& =-\int_{\sigma} \int_{-\infty}^{\sigma} \delta\left[\left(x_{1}-x_{2}\right)_{v}^{2}\right] j_{\mu}\left(x_{1}\right) \mathrm{d} \sigma_{\mu}\left(x_{1}\right) j_{m}\left(x_{2}\right) \mathrm{d} x_{2}-\int_{\sigma}^{\infty} \int_{\sigma} \delta\left[\left(x_{1}-x_{2}\right)_{v}^{2}\right] j_{\mu}\left(x_{2}\right) \mathrm{d} \sigma_{\mu}\left(x_{2}\right) j_{m}\left(x_{1}\right) \mathrm{d} x_{1} \\
& =-4 \pi \int_{\sigma} A_{m}^{\text {av. }}\left(x_{1}\right) j_{\mu}\left(x_{1}\right) \mathrm{d} \sigma_{\mu}\left(x_{1}\right)-\int_{\sigma} A_{m}^{\text {ret. }}\left(x_{2}\right) j_{\mu}\left(x_{2}\right) \mathrm{d} \sigma_{\mu}\left(x_{2}\right)=-i e 4 \pi \int \bar{\psi} \gamma_{v} \psi\left[A_{m}^{\text {av. }}+A_{m}^{\text {ret. }}\right] \mathrm{d} \sigma_{v} .
\end{aligned}
$$

Daher wird aus (11):

$$
G_{m}(\sigma)=\int_{\sigma} \bar{\psi} \gamma_{v} \psi_{\mid m} \mathrm{~d} \sigma_{v}+\frac{1}{8 \pi} \int_{\sigma}^{\infty} \int_{-\infty}^{\sigma} \delta_{\mid m}\left[\left(x_{1}-x_{2}\right)_{v}^{2}\right] j_{\mu}\left(x_{1}\right) j_{\mu}\left(x_{2}\right) \mathrm{d} x_{1} \mathrm{~d} x_{2} .
$$

${ }^{6}$ Der Faktor ${ }^{1 / 4}$ rührt her von der Berücksichtigung der doppelten Summation über das Raum-Zeit-Gebiet und vom Übergang von zwei Partikeln zu „einem“. 


\section{Qu antentheorie}

In der Quantentheorie verlangen wir zur Erreichung endlicher Selbstenergie, daß die Operatoren in der "richtigen“ ${ }^{2}$ Reihenfolge $\bar{\psi} \bar{\psi} \psi \psi$ stehen.

Wegen

$$
\frac{\partial}{\partial x_{v}}\left[\bar{\psi} \gamma_{v} \psi_{\mid m}\right]=\frac{i e}{2} \bar{\psi}\left[A_{m}^{\mathrm{av} .}+A_{m}^{\mathrm{ret} .}\right] \psi,
$$

was sich mit (7) leicht beweist, sieht man Gl.(13) unmittelbar an, daß im zweiten Integral von (13) die „richtige“ Reihenfolge nicht zu $\frac{\delta G}{\delta \sigma}=0$ führt. Wir können den Vektor (13) aber so umformen, daß sich diese Forderung erfüllen läßt.

Zunächst ist

$$
\int_{\sigma}^{\infty} \int_{-\infty}^{\sigma}=\int_{\sigma}^{\infty} \int_{-\infty}^{\infty}-\int_{\sigma}^{\infty} \int_{\sigma}^{\infty} .
$$

Der Integrand ist (nur klassisch!) antisymmetrisch in den Variablen $x_{1}$ und $x_{2}$. Für einen solchen Integranden aber wird

$\int_{\sigma}^{\infty} \int_{\sigma}^{\infty} J\left(x_{1}, x_{2}\right) \mathrm{d} x_{1} \mathrm{~d} x_{2}=-\int_{\sigma}^{\infty} \int_{\sigma}^{\infty} J\left(x_{2}, x_{1}\right) \mathrm{d} x_{2} \mathrm{~d} x_{1}=0$.

Daher wird aus (13):

$$
\begin{aligned}
G_{m}(\sigma) & =\int_{\sigma} \bar{\psi} \gamma_{v} \psi_{\mid m} \mathrm{~d} \sigma_{v} \\
& +\frac{1}{2} \int_{\sigma}^{\infty} i_{v}\left(x_{1}\right)\left[A_{v \mid m}^{\text {av. }}\left(x_{1}\right)+A_{\nu \mid m}^{\text {ret. }}\left(x_{1}\right)\right] \mathrm{d} x_{1} .
\end{aligned}
$$

Ebenso kann man einen entsprechenden Vektor mit

$-\int_{-\infty}^{\sigma}$ statt $+\int_{\sigma}^{\infty}$ herleiten. Die symmetrische Form ist dann

$$
\begin{aligned}
G_{m}(\sigma)= & \int_{\sigma} \bar{\psi} \gamma_{v} \psi_{\mid m} \mathrm{~d} \sigma_{v} \\
& +\frac{1}{4}\left\{\int_{\sigma}^{\infty}-\int_{-\infty}^{\sigma}\right\}\left[A_{v \mid m}^{\text {av. }}+A_{v \mid m}^{\text {ret. }}\right] i_{v}(x) \mathrm{d} x .
\end{aligned}
$$

Diese Form ist für die Quantentheorie brauchbar. Man schreibt nämlich hierfür:

$$
\begin{aligned}
& G_{m}(\sigma)=\int_{\sigma} \bar{\psi} \gamma_{v} \psi_{\mid m} \mathrm{~d} \sigma_{v} \\
& \quad+\frac{i e}{4}\left\{\int_{\sigma}^{\infty}-\int_{-\infty}^{\sigma}\right\} \bar{\psi}(x) \gamma_{v}\left[A_{v \mid m}^{\text {av. }}+A_{v \mid m}^{\text {ret. }}\right] \psi(x) \mathrm{d} x .
\end{aligned}
$$

Dieser Operator liefert die verlangte verschwindende Selbstenergie. Wendet man ihn nämlich auf den Zustand $\Phi_{1}$ („,ein Elektron“) an, so wird wegen (1):

$$
G_{m}(\sigma) \Phi_{1}=\int_{\sigma} \bar{\psi}_{0} \gamma_{v} \psi_{0 \mid m} \mathrm{~d} \sigma_{v} \Phi_{1}
$$

die Wechselwirkung mit $\operatorname{dem}$ Felde $A_{v}$, ist also nicht mehr enthalten.

Ich bin Hrn. Prof. Dr. L u dwig für die Anregung zu dieser Arbeit und zahlreiche fruchtbare und klärende Diskussionen sowie für die Ermöglichung dieser und damit zusammenhängender weiterer Untersuchungen zu besonderem Dank verpflichtet. Ich danke auch Hrn. G. S ü s s m a n n für kritische Bemerkungen und manchen Hinweis.

\title{
Über die Möglichkeit von Spinmodellen
}

\author{
Von Fritz Bopp und Rudolf HAAG $^{*}$ \\ Aus dem Institut für theoretische Physik der Universität München \\ (Z. Naturforschg. 5 a, 644—653 [1950]; eingegangen am 1. Dezember 1950)
}

\begin{abstract}
Es wird gezeigt, daß sich die Zustände des Spins in völliger Analogie zu denen anderer quantenmechanischer Größen durch stetige Eigenfunktionen gewisser Differentialoperatoren beschreiben lassen. Während die Kugelfunktionen mit halbzahligem Index hierfür bekanntlich wegen ihrer Transformationseigenschaften nicht in Frage kommen, besitzt bereits der Schrödingersche Drehimpulsoperator des Zweikörperproblems (echte) Eigenfunktionen zu halbzahligen Eigenwerten. Das Modell von Goudsmit-Uhlenbeck und das feldmechanische Modell des Spins werden unter diesem neuen Gesichtspunkt diskutiert.
\end{abstract}

E s ist eine weitverbreitete Ansicht, der Spin der Elementarteilchen sei grundsätzlich modellfremd, d. h. ein Phänomen, das im Bereich der klassischen Physik kein Analogon besitzt. Diese Meinung hat ihren Ursprung darin, daß der Schrödingersche Drehimpulsoperator nur ganzzahlige Eigenwerte liefert.
Die gelegentlich unternommenen Versuche, die Kugelfunktionen mit halbzahligem Index zur Darstellung des Spin-Phänomens heranzuziehen, sind gescheitert, und es schien, daß man zur Wiedergabe der halb-

* Vorgetragen auf der Tagung des Verbandes deutscher physikalischer Gesellschaften, Bad Nauheim, Oktober 1950. 